\title{
On cyclotomic schemes over finite near-fields
}

\author{
J. Bagherian • Ilia Ponomarenko • \\ A. Rahnamai Barghi
}

Received: 24 March 2006 / Accepted: 22 May 2007 /

Published online: 16 June 2007

(C) Springer Science+Business Media, LLC 2007

\begin{abstract}
We introduce a concept of cyclotomic association scheme over a finite near-field $\mathbb{K}$. It is proved that any isomorphism of two such nontrivial schemes is induced by a suitable element of the group $\operatorname{AGL}(V)$, where $V$ is the linear space associated with $\mathbb{K}$. A sufficient condition on a cyclotomic scheme $\mathcal{C}$ that guarantee the inclusion $\operatorname{Aut}(\mathcal{C}) \leq \operatorname{A} \Gamma L(1, \mathbb{F})$, where $\mathbb{F}$ is a finite field with $|\mathbb{K}|$ elements, is given.
\end{abstract}

Keywords Association scheme $\cdot$ Finite near-field · Permutation group

\section{Introduction}

An algebraic structure $\mathbb{K}=(\mathbb{K},+, \circ)$ is called a (right) near-field if $\mathbb{K}^{+}=(\mathbb{K},+)$ is a group with the neutral element $0_{\mathbb{K}}, \mathbb{K}^{\times}=\left(\mathbb{K} \backslash\left\{0_{\mathbb{K}}\right\}, \circ\right)$ is a group, $x \circ 0_{\mathbb{K}}=0_{\mathbb{K}}$ for all $x \in \mathbb{K}$, and

$$
(x+y) \circ z=x \circ z+y \circ z, \quad x, y, z \in \mathbb{K} .
$$

In the finite case, the group $\mathbb{K}^{+}$is elementary Abelian, and the group $\mathbb{K}^{\times}$is Abelian iff $\mathbb{K}$ is a field (as to near-fields theory, we refer to [13]). By the Zassenhaus theorem

I. Ponomarenko partially supported by RFFI, grants 03-01-00349, NSH-2251.2003.1.

J. Bagherian · A. Rahnamai Barghi $(\varangle)$

Institute for Advanced Studies in Basic Sciences (IASBS), P.O. Box 45195-1159, Zanjan, Iran e-mail: rahnama@iasbs.ac.ir

\section{J. Bagherian}

e-mail: bagherian@iasbs.ac.ir

\section{Ponomarenko}

Petersburg Department of V.A. Steklov Institute of Mathematics, Fontanka 27, St. Petersburg 191023, Russia

e-mail: inp@pdmi.ras.ru 
apart from seven exceptional cases, each finite near-field $\mathbb{K}$ is the Dickson near-field, i.e., there exist a finite field $\mathbb{F}_{0}$ and its extension $\mathbb{F}$ such that $\mathbb{F}^{+}=\mathbb{K}^{+}$and

$$
y \circ x=y^{\sigma_{x}} \cdot x, \quad x, y \in \mathbb{K},
$$

where $\sigma_{x} \in \operatorname{Aut}\left(\mathbb{F} / \mathbb{F}_{0}\right)$ and $\cdot$ denotes the multiplication in $\mathbb{F}$. In this case, $\left|\mathbb{F}_{0}\right|=q$ and $|\mathbb{K}|=|\mathbb{F}|=q^{n}$, where $q$ is a power of a certain prime $p$, and $n=\left[\mathbb{F}: \mathbb{F}_{0}\right]$. It can be proved that $(q, n)$ forms a Dickson pair, i.e., every prime factor of $n$ is a divisor of $q-1$ and $4 \mid n$ implies $4 \mid(q-1)$. There exist exactly $\varphi(n) / k$ nonisomorphic Dickson near-fields corresponding to the same Dickson pair $(q, n)$, where $k$ is the order of $p(\bmod n)$. The multiplicative group of any Dickson near-field is solvable (and even meta-cyclic).

Let $\mathbb{K}$ be a finite near-field and $K$ be a subgroup of the group $\mathbb{K}^{\times}$. Set $\mathcal{R}=$ $\left\{R_{a}\right\}_{a \in \mathbb{K}}$, where

$$
R_{a}=\left\{(x, y) \in \mathbb{K}^{2}: y-x \in a \circ K\right\} .
$$

Then it is easily seen that any element of $\mathcal{R}$ is a 2-orbit of the permutation group

$$
\Gamma(K, \mathbb{K})=\{x \mapsto x \circ b+c, x \in \mathbb{K}: b \in K, c \in \mathbb{K}\},
$$

and so the pair $(\mathbb{K}, \mathcal{R}$ ) forms an association scheme on $\mathbb{K}$ (see Sect. 2 for the background on permutation groups and association schemes). We call it the cyclotomic scheme over the near-field $\mathbb{K}$ and denote it by $\operatorname{Cyc}(K, \mathbb{K})$. The number $|K|$ is called the valency of the scheme. If $K=\mathbb{K}^{\times}$, then the scheme is of rank 2 , and we call it the trivial scheme. The set of all cyclotomic schemes of valency $m<q^{n}-1$ over a Dickson near-field corresponding to a Dickson pair $(q, n)$ is denoted by $\operatorname{Cyc}(q, n, m)$.

When $\mathbb{K}=\mathbb{F}$ is a field, we come to cyclotomic schemes introduced by P. Delsarte (1973), see [1, p. 66]. One can see that any two such schemes of the same valency are isomorphic. Moreover, the automorphism group of such a nontrivial scheme is a subgroup of the group $A \Gamma L(1, \mathbb{F})$ (see [1, p. 389]). However, there exist a number of cyclotomic schemes over near-fields which are not isomorphic to cyclotomic schemes over fields. The main purpose of this paper is to study isomorphisms of cyclotomic schemes over near-fields.

The additive group of a finite near-field $\mathbb{K}$ being an elementary Abelian one can be identified with the additive group of a linear space $V_{\mathbb{K}}$ over the prime field contained in the center of $\mathbb{K}$. The existence of an isomorphism between a cyclotomic scheme over a near-field $\mathbb{K}$ and a cyclotomic scheme over a near-field $\mathbb{K}^{\prime}$, implies that $|\mathbb{K}|=\left|\mathbb{K}^{\prime}\right|$ and hence that the linear spaces $V_{\mathbb{K}}$ and $V_{\mathbb{K}^{\prime}}$ are isomorphic. Thus to study isomorphisms of cyclotomic schemes, we can restrict ourselves to near-fields $\mathbb{K}$ with a fixed linear space $V=V_{\mathbb{K}}$.

Theorem 1.1 Let $\mathcal{C}$ and $\mathcal{C}^{\prime}$ be nontrivial cyclotomic schemes over near-fields $\mathbb{K}$ and $\mathbb{K}^{\prime}$, respectively. Suppose that $V=V_{\mathbb{K}}=V_{\mathbb{K}^{\prime}}$. Then $\operatorname{Iso}\left(\mathcal{C}, \mathcal{C}^{\prime}\right) \subset \operatorname{AGL}(V)$. In particular, $\operatorname{Aut}(\mathcal{C}) \leq \operatorname{AGL}(V)$.

For a trivial scheme $\mathcal{C}$, we obviously have $\operatorname{Aut}(\mathcal{C})=\operatorname{Sym}(\mathbb{K})$. Thus the inclusion $\operatorname{Aut}(\mathcal{C}) \leq \operatorname{AGL}(V)$ holds only if $|\mathbb{K}| \leq 4$. In general, the right-hand side of the first inclusion of Theorem 1.1 cannot be refined, because $\operatorname{Iso}(\mathcal{C}, \mathcal{C})=\operatorname{AGL}(V)$ for a 
scheme $\mathcal{C}=\operatorname{Cyc}(K, \mathbb{F})$, where $\mathbb{F}$ is a finite field of composite order, and $K$ is the multiplicative group of the prime subfield of $\mathbb{F}$.

We prove Theorem 1.1 in Sect. 3. The key ingredient for the proof is Theorem 3.2 showing that the operation of taking the 2-closure preserves the socle of any uniprimitive 3/2-transitive permutation groups of affine type. (Here we essentially use the result of [9].) From Theorem 1.1 we deduce a criterion for the isomorphism of cyclotomic schemes (Theorem 3.4).

The second part of Theorem 1.1 can be made much more precise in some cases. For instance, if the cyclotomic scheme $\mathcal{C}=\operatorname{Cyc}(K, \mathbb{K})$ is imprimitive, then $\operatorname{Aut}(\mathcal{C})=$ $\Gamma(K, \mathbb{K})$ (Corollary 3.5). In general, this equality does not hold even for a cyclotomic scheme over a finite field, because the group $\operatorname{Aut}(\mathcal{C})$ can contain some automorphisms of this field. However, we are able to specify the automorphisms of a cyclotomic scheme by using Zsigmondy prime divisors of its valency.

Definition 1.2 Given integers $q, n \in \mathbb{N}$, a prime divisor $r$ of $q^{n}-1$ is called a Zsigmondy prime for $(q, n)$ if $r$ does not divide $q^{i}-1$ for all $1 \leq i<n$. The set of all such primes greater than a fixed number $k \in \mathbb{N}$ is denoted by $Z_{k}(q, n)$.

It is known that at least one Zsigmondy prime for $(q, n)$ exists unless $(q, n)=$ $(2,6)$, or $q+1$ is a power of 2 and $n=2$ (see, e.g., [11]). Moreover, any such prime is of the form $r=a n+1$ for some $a \geq 1$.

Theorem 1.3 Let $\mathcal{C} \in \operatorname{Cyc}\left(p^{d}, n, m\right)$ be a cyclotomic scheme over a Dickson nearfield and $k=d n$. Then $\operatorname{Aut}(\mathcal{C}) \leq \mathrm{A} \Gamma \mathrm{L}\left(1, p^{k}\right)$ whenever $m$ has a prime divisor $r \in$ $Z_{2 k+1}(p, k)$.

From Lemma 4.2 it follows that for a fixed $p^{d}$ the set $Z_{2 k+1}(p, k)$ is not empty for all sufficiently large $k$. This fact enables us to prove that the hypothesis of Theorem 1.3 is satisfied in many cases. More precisely, the following statement holds.

Theorem 1.4 Let $\mathcal{C} \in \operatorname{Cyc}\left(p^{d}, n, m\right)$ be a cyclotomic scheme over a Dickson near-field and $q=p^{d}$. Then $\operatorname{Aut}(\mathcal{C}) \leq \mathrm{A} \Gamma \mathrm{L}\left(1, q^{n}\right)$ for all $n \gg q$ such that $\left|Z_{2 d n+1}(p, d n)\right| \neq 1$.

Theorem 1.4 is proved in Sect. 4 by means of the classification of linear groups with orders having certain large prime divisors given in [4]. We believe that a more delicate analysis of this classification could improve our result to show that given a prime power $q$ for all but finitely many Dickson pairs $(q, n)$, the inclusion $\operatorname{Aut}(\mathcal{C}) \leq$ $\mathrm{A} \Gamma \mathrm{L}\left(1, q^{n}\right)$ holds for all nontrivial cyclotomic schemes $\mathcal{C}$ over a Dickson near-field corresponding to $(q, n)$.

\section{Permutation groups and association schemes}

\section{1}

Concerning basic facts of finite permutation group theory, we refer to [3]. Let $V$ be a finite set, $\Gamma \leq \operatorname{Sym}(V)$, and $m \in \mathbb{N}$. Denote by $\operatorname{Orb}_{m}(\Gamma)$ the set of all orbits of the 
induced action of $\Gamma$ on the set $V^{m}$; these orbits are called the $m$-orbits of $\Gamma$. The largest subgroup of $\operatorname{Sym}(V)$ the $m$-orbits of which coincide with those of $\Gamma$ is called the $m$-closure of $\Gamma$; we denote it by $\Gamma^{(m)}$.

Let $U$ be a set with at least two elements, and let $m \geq 2$ be an integer. Following [9], we say that a permutation group $G \leq \operatorname{Sym}(V)$ preserves a product decomposition $U^{m}$ of $V$ if the latter can be identified with the Cartesian product $U^{m}$ in such a way that $G$ is a subgroup of the wreath product $\operatorname{Sym}(U)$ ? $\operatorname{Sym}(m)$ in product action. Any element $g$ of the latter group induces uniquely determined permutations $g_{1}, \ldots, g_{m} \in$ $\operatorname{Sym}(U)$ and $\sigma \in \operatorname{Sym}(m)$ such that

$$
\left(u_{1}, \ldots, u_{m}\right)^{g}=\left(u_{i_{1}}^{g_{i_{1}}}, \ldots, u_{i_{m}}^{g_{i_{m}}}\right), \quad \text { where } i_{j}=j^{\sigma^{-1}} .
$$

If $G$ projects onto a transitive subgroup of $\operatorname{Sym}(m)$, then the subgroup of index $m$ in $G$ stabilizing the first entry of points of $U^{m}$ induces a subgroup of $\operatorname{Sym}(U)$ by permuting the first entries of points of $V=U^{m}$; this subgroup is called the group induced by $G$ on $U$. The following statement being a special case of result [9, Lemma 4.1] will be used in Sect. 3. Below a primitive group is called uniprimitive if it is not 2-transitive, and it is called of affine type if its socle is Abelian.

Theorem 2.1 Let $G \leq \operatorname{Sym}(V)$ be a uniprimitive group of affine type. Suppose that $\operatorname{soc}(G) \neq \operatorname{soc}\left(G^{(2)}\right)$. Then $G$ and $G^{(2)}$ preserve a product decomposition $V=U^{m}$ such that $|U| \geq 5, m \geq 2$, and the group induced by $G^{(2)}$ on $U$ contains $\operatorname{Alt}(U)$.

Let $V$ be a finite set and $\mathcal{R}$ a partition of the set $V^{2}$ containing its diagonal $\Delta(V)$ and closed with respect to the permutation of coordinates. The pair $\mathcal{C}=(V, \mathcal{R})$ is called an association scheme or a scheme on $V$ if, given binary relations $R, S, T \in \mathcal{R}$, the number

$$
|\{v \in V:(u, v) \in R,(v, w) \in S\}|
$$

does not depend on the choice of $(u, w) \in T$. The elements of $\mathcal{R}$ and the number $|\mathcal{R}|$ are called the basis relations and the rank of $\mathcal{C}$ respectively. The scheme is called imprimitive if a union of some of its basis relations is an equivalence relation on $V$ other than $\Delta(V)$ and $V^{2}$; otherwise the scheme is called primitive whenever $|V|>1$.

Two schemes $\mathcal{C}=(V, \mathcal{R})$ and $\mathcal{C}^{\prime}=\left(V^{\prime}, \mathcal{R}^{\prime}\right)$ are called isomorphic if there exists a bijection $f: V \rightarrow V^{\prime}$, called the isomorphism from $\mathcal{C}$ to $\mathcal{C}^{\prime}$, such that $\mathcal{R}^{f}=\mathcal{R}^{\prime}$, where $\mathcal{R}^{f}=\left\{R^{f}: R \in \mathcal{R}\right\}$ with $R^{f}=\left\{\left(u^{f}, v^{f}\right):(u, v) \in R\right\}$. The set of all such isomorphisms is denoted by $\operatorname{Iso}\left(\mathcal{C}, \mathcal{C}^{\prime}\right)$. The group $\operatorname{Iso}(\mathcal{C})=\operatorname{Iso}(\mathcal{C}, \mathcal{C})$ contains the normal subgroup

$$
\operatorname{Aut}(\mathcal{C})=\left\{g \in \operatorname{Sym}(V): R^{g}=R, R \in \mathcal{R}\right\}
$$

called the automorphism group of the scheme $\mathcal{C}$.

A wide class of schemes comes from permutation groups as follows. Let $\Gamma \leq$ $\operatorname{Sym}(V)$ be a permutation group and $\mathcal{R}=\operatorname{Orb}_{2}(\Gamma)$. Then the pair $\operatorname{Inv}(\Gamma)=(V, \mathcal{R})$ is a scheme and

$$
\operatorname{Aut}(\operatorname{Inv}(\Gamma))=\Gamma^{(2)}
$$


In particular, any cyclotomic scheme $\operatorname{Cyc}(K, \mathbb{K})$ over a near-field $\mathbb{K}$ equals the scheme $\operatorname{Inv}(\Gamma)$ with $\Gamma=\Gamma(K, \mathbb{K})$ (see (4)). One can prove that this scheme is primitive iff so is the group $\Gamma$.

\section{3}

Let $\mathbb{K}$ be a near-field and $K \leq \mathbb{K}^{\times}$. Then the group $\Gamma(K, \mathbb{K})$ defined by (4) can be naturally identified with a subgroup of the group $\operatorname{AGL}(V)$, where $V=V_{\mathbb{K}}$ (see Sect. 1). Under this identification, the group $K$ (considered as a subgroup of the group $\Gamma(K, \mathbb{K}))$ goes to a subgroup of the group $\mathrm{GL}(V)$. This subgroup is called the base group of the cyclotomic scheme $\operatorname{Cyc}(K, \mathbb{K})$.

Theorem 2.2 Let $\mathcal{C}$ be a cyclotomic scheme over a near-field $\mathbb{K}$. Then $\mathcal{C}$ is primitive iff the base group of $\mathcal{C}$ is irreducible.

Proof Let $\mathcal{C}=\operatorname{Cyc}(K, \mathbb{K})$ for some group $K \leq \mathbb{K}^{\times}$. Then the scheme $\mathcal{C}$ is primitive iff the group $\Gamma=\Gamma(K, \mathbb{K})$ is primitive (see Subsect. 2.2). However, from [3, Theorem 4.7.A] it follows that the latter statement holds iff the stabilizer of the point $0_{\mathbb{K}}$ in the group $\Gamma$ is an irreducible subgroup of the group $\operatorname{GL}\left(V_{\mathbb{K}}\right)$. Since this stabilizer coincides with the base group of the scheme $\mathcal{C}$, we are done.

It should be noted that the base group of a primitive cyclotomic scheme $\mathrm{Cyc}(K, \mathbb{K})$ can be primitive (as a linear group) or not. For example, it is always primitive for $K=\mathbb{K}^{\times}$, and it is imprimitive for $K=\{1\}$ if the number $|\mathbb{K}|$ is a composite one.

Corollary 2.3 The cyclotomic scheme $\mathcal{C}$ in Theorem 1.3 is primitive.

Proof Let $G$ be the base group of the scheme $\mathcal{C}$. Then $G$ is a solvable subgroup of the group $\operatorname{GL}(k, p)$, and $r$ divides the order $m$ of $G$. By [6, Proposition 6.3] this implies that the group $G$ is irreducible. Thus the scheme $\mathcal{C}$ is primitive by Theorem 2.2.

Let $V$ be a finite dimensional linear space over a finite prime field, and let $G \leq$ $\mathrm{GL}(V)$ be an irreducible Abelian group. Then $G$ is a cyclic group and its linear span $L(G)$ in the algebra $\operatorname{End}(V)$ is a finite field with $|V|$ elements (see [6, Lemma 0.5]). The multiplicative group of this field acts regularly on nonzero vectors of $V$, i.e., this group is a Singer subgroup of the group $\operatorname{GL}(V)$. So, given a fixed nonzero $u_{0} \in V$, the mapping

$$
\tau: L(G) \rightarrow V, \quad A \mapsto A u_{0},
$$

is a bijection. This defines a field $\mathbb{F}=\mathbb{F}(G)$ with elements from $V$ such that $\mathbb{F}^{+}$ coincides with the additive group of the linear space $V$. Clearly, $\tau(G) \leq \mathbb{F}^{\times}$.

Theorem 2.4 Any primitive cyclotomic scheme with Abelian base group is a cyclotomic scheme over a field.

Proof Let $\mathcal{C}=\operatorname{Cyc}(K, \mathbb{K})$ be a primitive cyclotomic scheme and $V=V_{\mathbb{K}}$. Suppose that its base group $G \leq \mathrm{GL}(V)$ is Abelian. Then $G$ is irreducible by Theorem 2.2. 
This enables us to construct the field $\mathbb{F}=\mathbb{F}(G)$. From the definition of this field it follows that $\mathbb{F}^{+}=\mathbb{K}^{+}$and

$$
x \circ y=x \cdot y, \quad x \in V, y \in M,
$$

where $M=\tau(G)$, and $\circ$ and $\cdot$ denote the multiplications in $\mathbb{K}$ and $\mathbb{F}$, respectively. This implies that $\Gamma(K, \mathbb{K})=\Gamma(M, \mathbb{F})$, and hence $\mathcal{C}=\operatorname{Cyc}(M, \mathbb{F})$ is a cyclotomic scheme over the field $\mathbb{F}$.

\section{An isomorphism criterion for cyclotomic schemes}

\section{1}

In this section, we prove Theorem 1.1. For cyclotomic schemes with primitive base group we will use Theorem 2.1. In the imprimitive case, we need an auxiliary result on 3/2-transitive groups, where by such a group we mean a transitive permutation group $\Gamma$ for which the orbits of its one point stabilizer $\Gamma_{v}$ other than $\{v\}$ all have the same size.

Lemma 3.1 Let $G \leq \operatorname{Sym}(V)$ be a 3/2-transitive group preserving a product decomposition $V=U^{m}$ for $m \geq 2$. Then the stabilizer $G_{u, v}$ of some points $u, v \in V$ is an Abelian 2-group.

Proof Let $u \in V$ and $I=\{1, \ldots, m\}$. Without loss of generality we may assume that $u=\left(u_{0}, \ldots, u_{0}\right) \in U^{m}$ for some $u_{0} \in U$. Then from (5) it follows that $u_{0}^{g_{i}}=u_{0}$ for all $g \in G_{u}$ and all $i \in I$. So the cardinality of the set $I_{v}=\left\{i \in I: v_{i} \neq u_{0}\right\}$, where $v_{i}$ is the $i$ th component of $v \in V$, does not depend on the choice of $v$ inside of an orbit of the group $G_{u}$. Thus, the sets

$$
\begin{aligned}
V_{k} & =\left\{v \in V:\left|I_{v}\right|=k\right\}, \quad k=1,2, \\
R & =\left\{(v, w) \in V_{1} \times V_{2}: v_{i}=w_{i} \text { for the unique } i \in I_{v}\right\}
\end{aligned}
$$

are $G_{u}$-invariant. Obviously, $\left|R_{\text {in }}(w)\right|=2$ for all $w \in V_{2}$ where $R_{\text {in }}(w)=\{v \in V$ : $(v, w) \in R\}$. We divide the remaining argument into a sequence of claims.

Claim 1 Let $X \in \operatorname{Orb}\left(G_{u}, V_{1}\right), Y \in \operatorname{Orb}\left(G_{u}, V_{2}\right)$, and $S=R \cap(X \times Y)$. Then

$$
\left|S_{\text {out }}(x)\right| \leq 2, \quad x \in X,
$$

where $S_{\text {out }}(x)=\{v \in V:(x, v) \in S\}$. Indeed, since $S$ is a $G_{u}$-invariant relation, the numbers $\left|S_{\text {out }}(x)\right|$ and $\left|S_{\text {in }}(y)\right|$ do not depend on $x \in X$ and $y \in Y$, respectively. If we denote them by $a$ and $b$, then obviously $|X| a=|Y| b$. Taking into account that $|X|=|Y|$ due to 3/2-transitivity of $G$, we conclude that $a=b \leq 2$ (see the above remark). 
Claim 2 Let $x$ and $y$ be elements of $V_{1}$ such that $I_{x} \neq I_{y}$. Then

$$
\left|y^{G_{u, x}}\right| \leq 2
$$

Indeed, let $I_{x}=\{i\}$ and $I_{y}=\{j\}$ for some distinct $i, j \in I$. Then there exists a uniquely determined element $w \in V_{2}$ such that $x_{i}=w_{i}$ and $y_{j}=w_{j}$. Denote by $X$ and $Y$ the orbits of the group $G_{u}$ containing $x$ and $w$, respectively. From Claim 1, it follows that $S_{\text {out }}(x)=\left\{w, w^{\prime}\right\}$ for some $w^{\prime} \in Y$. Since the set $S_{\text {out }}(x)$ is obviously $G_{u, x}$-invariant, we conclude that so is the set $R_{\text {in }}(w) \cup R_{\text {in }}\left(w^{\prime}\right)$. However, this set contains at most three elements two of which are $x$ and $y$. Thus

$$
\left|y^{G_{u, x}}\right| \leq\left|\left(R_{\mathrm{in}}(w) \cup R_{\mathrm{in}}\left(w^{\prime}\right)\right) \backslash\{x\}\right| \leq 2,
$$

which proves the claim.

Claim 3 Let $(x, w) \in V_{1} \times V_{2}$. Then the transitive constituent $H$ of the group $G_{u, x}$ induced by its action on the set $Y=w^{G_{u, x}}$ is a 2-group. Indeed, without loss of generality we may assume that $|Y|>2$ and $I_{w}=\{i, j\}$ for some distinct $i, j \in I$. Then $i, j \notin I_{x}$, since otherwise $Y \subset R_{\text {out }}(x)$ and hence $|Y| \leq 2$ by Claim 1 . Set $y$ to be the unique element of $V_{1} \backslash\{x\}$ such that $y_{i}=w_{i}$. By Claim 2 the set $X=y^{G_{u, x}}$ consists of (not necessary distinct) elements $y, z \in V_{1}$, whence by Claim 1 it follows that

$$
Y=S_{\text {out }}(y) \cup S_{\text {out }}(z), \quad 1 \leq\left|S_{\text {out }}(y)\right|=\left|S_{\text {out }}(z)\right| \leq 2 .
$$

Since $|Y|>2$ and $\left|S_{\text {in }}(w)\right|=\left|S_{\text {in }}\left(w^{\prime}\right)\right|$ for all $w^{\prime} \in Y$, we conclude that $S_{\text {out }}(y)$ and $S_{\text {out }}(z)$ are disjoint blocks of the group $H$, and each of them is of size 2. This implies that $H$ is a 2-group isomorphic to a subgroup of the group $\operatorname{Sym}(2)$ 2 $\operatorname{Sym}(2)$.

Claim 4 The action of $G_{u}$ on $V_{2}$ is faithful. Indeed, any $g \in G_{u}$ is of the form (5). Suppose that $w^{g}=w$ for all $w \in V_{2}$. Then, given $i \in I$ and all $w \in V_{2}$ such that $\{i, j\} \subset I_{w}$ and $w_{i}=w_{j}$ where $j=i^{\sigma}$, we have

$$
w_{j}=\left(w_{i_{j}}\right)^{g_{i_{j}}}=\left(w_{i}\right)^{g_{i}}=\left(w_{j}\right)^{g_{i}} .
$$

This implies that $g_{i}=\mathrm{id}_{U}$ for all $i \in I$. Next, if $\sigma \neq \mathrm{id}_{I}$, then obviously $w^{g} \neq w$ for all $w \in V_{2}$ such that $I_{w}=\{i, j\}$ and $w_{i} \neq w_{j}$, where $j=i^{\sigma}$. Thus $g=\mathrm{id}_{V}$, and we are done.

To complete the proof of Lemma 3.1 take $v \in V_{1}$. Denote by $K$ the direct product of transitive constituents of the group $G_{u, v}$ corresponding to its orbits contained in the set $V_{2}$. Then $K$ is a 2-group by Claim 3. On the other hand, by Claim 4 the group $G_{u, v}$ is isomorphic to a subgroup of the group $K$. Thus $G_{u, v}$ is a 2-group.

Theorem 3.2 Let $G \leq \operatorname{Sym}(V)$ be a uniprimitive 3/2-transitive group of affine type. Then $\operatorname{soc}(G)=\operatorname{soc}(\Gamma)$, where $\Gamma=G^{(2)}$.

Proof Suppose that $\operatorname{soc}(G) \neq \operatorname{soc}(\Gamma)$. Then from Theorem 2.1 it follows that the groups $G$ and $\Gamma$ preserve a product decomposition $V=U^{m}$ such that $|U| \geq 5$, 
$m \geq 2$, and the group induced by $\Gamma$ on $U$ contains $\operatorname{Alt}(U)$. This implies that

$$
|\Gamma|=\operatorname{am}|\operatorname{Alt}(U)|
$$

for some $a \in \mathbb{N}$. On the other hand, the group $\Gamma$ obviously is 3/2-transitive. Denote by $d$ the size of an orbit of its one point stabilizer $\Gamma_{v}$ other than $\{v\}$. Then it is easy to see that $d=m e$ for some divisor $e$ of $|U|-1$ (it suffices to check the orbit of a point from the set $V_{1}$ defined in (6)). By Lemma 3.1 for $G=\Gamma$ this implies that

$$
|\Gamma|=|V| m e 2^{k}
$$

for some $k \in \mathbb{N}$. Thus equalities (7) and (8) show that $|\operatorname{Alt}(U)|$ divides $|V| e 2^{k}$. Since $e$ divides $|U|-1$, it follows that $(|U|-2)$ ! divides $|V| 2^{k+1}$. However, this is impossible for $|U| \geq 5$, since $|V|$ is a prime power (we used the fact that $G$ is of affine type).

From Theorem 3.2 it follows that $G^{(2)}$ is a uniprimitive 3/2-transitive group of affine type. If in addition, the group $G$ preserves a product decomposition, then the same decomposition is preserved by $G^{(2)}$. Thus, in this case, the form of this group can be found by means of the classification of 3/2-transitive imprimitive linear groups given in [8].

\section{2}

In this subsection we fix a near-field $\mathbb{K}$ and a cyclotomic scheme $\mathcal{C}$ over $\mathbb{K}$ and denote by $T=T_{V}$ the translation group of the linear space $V=V_{\mathbb{K}}$. Clearly, $T \leq \operatorname{Sym}(V)$.

Lemma 3.3 If the scheme $\mathcal{C}$ is nontrivial, then $T$ is a characteristic subgroup of the group $\operatorname{Aut}(\mathcal{C})$. More exactly, the following statements hold:

(1) If $\mathcal{C}$ is imprimitive, then $\operatorname{Aut}(\mathcal{C})$ is a Frobenius group with kernel $T$.

(2) If $\mathcal{C}$ is primitive, then $T=\operatorname{soc}(\operatorname{Aut}(\mathcal{C}))$.

Proof Let $\mathcal{C}=\operatorname{Cyc}(K, \mathbb{K})$ and $\Gamma=\Gamma(K, \mathbb{K})$, where $K<\mathbb{K}^{\times}$(see (4)). Then $\mathcal{C}=$ $\operatorname{Inv}(\Gamma)$ and so $\operatorname{Aut}(\mathcal{C})=\Gamma^{(2)}$. On the other hand, it is easy to see that the orbits of the group $\Gamma_{v}$ other than $\{v\}$ all have the same size $|K|$. This implies that the group $\Gamma$ and hence the group $\operatorname{Aut}(\mathcal{C})$ is 3/2-transitive.

Let $\mathcal{C}$ be an imprimitive scheme. Then the group $\operatorname{Aut}(\mathcal{C})$ is imprimitive. Since any 3/2-transitive group is either primitive or a Frobenius group [14, Theorem 10.4], it follows that $\operatorname{Aut}(\mathcal{C})$ is a Frobenius group. The kernel of this group is of order $|V|=|T|$ and contains all fixed-point-free elements of the group $\Gamma$. Thus the kernel coincides with $T$, which proves statement (1).

Let $\mathcal{C}$ be a primitive scheme. Then the group $\Gamma$ is primitive and $T$ is a normal Abelian subgroup of it. This implies that the socle of $\Gamma$ is Abelian and hence coincides with $T$ (see [3, Theorem 4.3.B]). Thus $\Gamma$ is a uniprimitive 3/2-transitive group of affine type. By Theorem 3.2 this implies that

$$
T=\operatorname{soc}(\Gamma)=\operatorname{soc}\left(\Gamma^{(2)}\right)=\operatorname{soc}(\operatorname{Aut}(\mathcal{C})),
$$

which completes the proof of the lemma. 
Proof of Theorem 1.1 Let $f \in \operatorname{Iso}\left(\mathcal{C}, \mathcal{C}^{\prime}\right)$. Then the bijection $f$ induces an isomorphism between permutation groups $\operatorname{Aut}(\mathcal{C})$ and $\operatorname{Aut}\left(\mathcal{C}^{\prime}\right)$. Since these groups are transitive, without loss of generality we may assume that $f$ leaves the point $0 \in V$ fixed. Then it suffices to verify that $f$ belongs to the group $\operatorname{Aut}(T)=\operatorname{GL}(V)$. However, the schemes $\mathcal{C}$ and $\mathcal{C}^{\prime}$ and hence the groups $\operatorname{Aut}(\mathcal{C})$ and $\operatorname{Aut}\left(\mathcal{C}^{\prime}\right)$ are primitive or not simultaneously. Thus the required statement follows from Lemma 3.3.

\section{3}

To make the statements of Theorem 1.1 more precise, given a group $G \leq \mathrm{GL}(V)$, we set

$$
\bar{G}=G^{(1)} \cap \mathrm{GL}(V) .
$$

Clearly, $\bar{G}$ coincides with the largest group $H \leq \mathrm{GL}(V)$ such that $\operatorname{Orb}(H)=\operatorname{Orb}(G)$.

Theorem 3.4 Under the conditions of Theorem 1.1, denote by $G$ and $G^{\prime}$ the base groups of the schemes $\mathcal{C}$ and $\mathcal{C}^{\prime}$, respectively. Then these schemes are isomorphic iff the groups $\bar{G}$ and $\overline{G^{\prime}}$ are conjugate in $\operatorname{GL}(V)$. Moreover, $\operatorname{Aut}(\mathcal{C})=T \bar{G}$.

Proof The first part of the theorem follows from the second one. Indeed, set $\Gamma=$ $\operatorname{Aut}(\mathcal{C})$ and $\Gamma^{\prime}=\operatorname{Aut}\left(\mathcal{C}^{\prime}\right)$. Then by Theorem 1.1 the schemes $\mathcal{C}$ and $\mathcal{C}^{\prime}$ are isomorphic iff there exists $g \in \mathrm{GL}(V)$ such that $g^{-1} \Gamma g=\Gamma^{\prime}$ or, equivalently, that $g^{-1} \Gamma_{v} g=\Gamma_{v}^{\prime}$ where $v$ is the zero vector of the linear space $V$. Since by the second part $\Gamma_{v}=\bar{G}$ and $\Gamma_{v}^{\prime}=\overline{G^{\prime}}$, we are done.

To prove the second part of the theorem we note that from Theorem 1.1 it follows that $\Gamma=T \Gamma_{v}$ and $\Gamma_{v} \leq \mathrm{GL}(V)$. Since obviously $\operatorname{Orb}\left(\Gamma_{v}\right)=\operatorname{Orb}(G)$, we conclude that $\Gamma_{v} \leq G^{(1)}$ and $\operatorname{Orb}\left(\Gamma_{v}\right)=\operatorname{Orb}(\bar{G})$. This shows that $\operatorname{Orb}_{2}(\Gamma)=\operatorname{Orb}_{2}(T \bar{G})$, whence by maximality of the 2 -closure it follows that $T \bar{G} \leq \Gamma$. Thus $\operatorname{Aut}(\mathcal{C})=\Gamma=$ $T \bar{G}$, and we are done.

For imprimitive cyclotomic schemes, Theorem 3.4 can be slightly simplified. Indeed, in this case, $\operatorname{Aut}(\mathcal{C})$ is a Frobenius group by statement (1) of Lemma 3.3. So

$$
|\bar{G}|=\left|\operatorname{Aut}(\mathcal{C})_{v}\right|=|X|=|G|
$$

where $X$ is an orbit of the group $\operatorname{Aut}(\mathcal{C})_{v}$ other than $\{v\}$. Since also $G \leq \bar{G}$, we have $\bar{G}=G$. Thus by Theorem 3.4 we obtain the following statement.

Corollary 3.5 Let the cyclotomic schemes $\mathcal{C}$ and $\mathcal{C}^{\prime}$ be imprimitive. Then they are isomorphic iff their base groups are conjugate in $\mathrm{GL}(V)$. Moreover, $\bar{G}=G$ and $\operatorname{Aut}(\mathcal{C})=T G$.

\section{Proof of Theorems 1.3 and 1.4}

The main tool of this section is Theorem 4.1 below which is deduced from the classification of linear groups with orders having certain large prime divisors [4]. In our 
case such a divisor is a Zsigmondy prime $r$ for a pair $(q, n)$, where $q$ is a prime power and $n \in \mathbb{N}$. Any cyclic group $G \leq \operatorname{GL}(n, q)$ of order $r$ is irreducible [6, Proposition 6.3], and the linear span $L(G)$ of it in $\operatorname{Mat}(n, q)$ is a finite field $\mathbb{F}$ with $q^{n}$ elements. We will identify the group $\Gamma \mathrm{L}(1, \mathbb{F})$ with a subgroup of $\operatorname{GL}(n, q)$. Below a group $\Gamma \leq \operatorname{GL}(n, q)$ is called half-transitive if the action of it on the set $V^{*}$ of nonzero vectors in the underlying linear space is intransitive and the orbits of this action all have the same size.

Theorem 4.1 Let $G \leq \Gamma \leq \operatorname{GL}(n, q)$ where $(q, n) \notin\{(2,4),(2,6)\}$. Suppose that $G$ is a cyclic group of order $r \in Z_{2 n+1}(q, n)$ and that the group $\Gamma$ is half-transitive. Then $\Gamma \leq \Gamma \mathrm{L}(1, \mathbb{F})$, where $\mathbb{F}=L(G)$.

Proof It suffices to prove that $\Gamma \leq \Gamma \mathrm{L}\left(1, q^{n}\right)$. Indeed, in this case $\Gamma \leq \Gamma \mathrm{L}\left(1, \mathbb{F}^{\prime}\right)$ for some field $\mathbb{F}^{\prime} \subset \operatorname{Mat}(n, q)$ with $q^{n}$ elements. So the multiplicative group of $\mathbb{F}^{\prime}$ normalizes $G$ and hence normalizes the Singer subgroup $\mathbb{F}^{\times} \subset L(G)$ of the group $\operatorname{GL}(n, q)$. However, the normalizer of $\mathbb{F}^{\times}$in $\operatorname{GL}(n, q)$ contains the unique Singer subgroup [2, Proposition 2.5]. This proves that $\mathbb{F}^{\prime}=\mathbb{F}$.

Suppose that $\Gamma$ is a solvable group. If $r$ divides the order of the Fitting subgroup of $\Gamma$, then this group is isomorphic to a subgroup of $\Gamma \mathrm{L}\left(1, q^{n}\right)[6$, Lemma 6.4]. Otherwise from Lemma 6.7 of the same book it follows that $r=n+1$, which contradicts the hypothesis on $r$. Thus the required statement is true for solvable groups. In particular, we may assume that $n \geq 2$ and that the group $\Gamma$ is nonsolvable.

Let $n=2$. From the classification of all subgroups of $\operatorname{GL}(2, q)$ given in [7, Proposition 8.1] it follows that any nonsolvable irreducible subgroup of $\operatorname{GL}(2, q)$, say $\Gamma$, has a subgroup $H$ such that $[\Gamma: H]$ divides $q-1$ and

$$
H \geq \operatorname{SL}\left(2, q^{\prime}\right) \quad \text { or } \quad H / Z \cong \operatorname{Alt}(5),
$$

where $q^{\prime} \geq 5$ is a divisor of $q$, and $Z$ is the subgroup of scalar matrices contained in $H$. However, in the former case, this group acts transitively on the set $V^{*}$, and the intransitivity of $\Gamma$ gives a contradiction. In the latter case, the prime divisors of the number $|\Gamma|$ are over those of the number $(q-1) 5 ! / 2$, which contradicts the assumption that $r \in Z_{2 n+1}(q, n)$ for $n=2$. It should be mentioned that in this case we proved the required statement for the group $\Gamma$ which is intransitive but not necessary half-transitive.

Let $n \geq 3$. The Zsigmondy prime $r$ for the pair $(q, n)$ is a primitive prime divisor of $q^{n}-1$ in the sense of [4]. Since $r$ divides the order of the group $\Gamma$, this group satisfies the hypothesis of the Main Theorem of that paper for $d=e=n$. In this case, the Main Theorem shows that, for the group $\Gamma$ (not necessary half-transitive) and $r>2 n+1$, one of the following statements holds:

(1) $\Gamma$ has a normal subgroup $\Gamma^{\prime}$ isomorphic to one of the classical groups $\operatorname{SL}\left(n, q^{\prime}\right)$, $\operatorname{Sp}\left(n, q^{\prime}\right), \operatorname{SU}\left(n, q^{\prime 1 / 2}\right)$, or $\Omega^{\epsilon}\left(n, q^{\prime}\right)$, where $r$ divides the order of $\Gamma^{\prime}, q^{\prime}$ is the order of a subfield of the ground field, and $\epsilon \in\{0,+,-\}$,

(2) $\Gamma \leq \mathrm{GL}\left(n / m, q^{m}\right) \cdot m$, and the number $r$ divides the order of the group $\Gamma \cap \mathrm{GL}\left(n / m, q^{m}\right)$, where $m$ is a divisor of $n$ other than 1 ,

(3) $(q, n)=(2,4)$ or $(2,6)$, 
where $\operatorname{GL}\left(n / m, q^{m}\right) \cdot m$ is the general linear group $\operatorname{GL}\left(n / m, q^{m}\right)$ embedded to $\operatorname{GL}(n, q)$ and extended by the group of automorphisms of the field extension $\mathrm{GF}\left(q^{m}\right) / \mathrm{GF}(q)$. However, the case (3) does not arise by the hypothesis of the theorem. Let us prove that the same is true in the other two cases.

We claim that the group $\Gamma$ contains a normal nonsolvable subgroup $H_{0}$ isomorphic to one of the groups $\mathrm{SL}\left(n_{0}, q_{0}\right), \mathrm{Sp}\left(n_{0}, q_{0}\right), \mathrm{SU}\left(n_{0}, q_{0}^{1 / 2}\right)$, or $\Omega^{\epsilon}\left(n_{0}, q_{0}\right)$, where $r$ divides the order of $H_{0}, n_{0} \geq 2$ is a divisor of $n$, and $q_{0}$ is the order of a subfield of the field $\operatorname{GF}\left(q^{n / n_{0}}\right)$. Indeed, in case (1) we can take $H_{0}=\Gamma^{\prime}$ and $\left(n_{0}, q_{0}\right)=\left(n, q^{\prime}\right)$. Otherwise, case (2) holds. It is easy to see that $\Gamma_{0}=\Gamma \cap \mathrm{GL}\left(n / m, q^{m}\right)$ is a normal subgroup of $\Gamma$ and $\Gamma / \Gamma_{0}$ is a cyclic group of order coprime to $r$. This implies that the group $\Gamma$ has a characteristic subgroup $H \leq \Gamma_{0}$ such that the factor group $\Gamma / H$ is solvable and each prime divisor of its order divides $m$. In particular,

$$
G \leq H \leq \mathrm{GL}\left(n / m, q^{m}\right)
$$

and $H$ is solvable iff $\Gamma$ is so. If case (2) holds for the group $H$, we repeat this argument with $\Gamma=H$ and $(n, q)=\left(n / m, q^{m}\right)$. Finally, we find a nonsolvable characteristic subgroup $H$ of the group $\Gamma$ such that $G \leq H \leq \operatorname{GL}\left(n_{1}, q_{1}\right)$, where $n_{1} \geq 2$ is a divisor of $n$, and $q_{1}$ is the order of a subfield of the field $\operatorname{GF}\left(q^{n / n_{1}}\right)$. Moreover, we may assume that case (1) holds for $\Gamma=H$ and $(n, q)=\left(n_{1}, q_{1}\right)$. Since the corresponding classical group $\Gamma^{\prime}$ is a characteristic subgroup of $H$, we are done with $H_{0}=\Gamma^{\prime}$ and $\left(n_{0}, q_{0}\right)=\left(n_{1}, q_{1}^{\prime}\right)$.

To complete the proof we will show that the above claim contradicts the halftransitivity of $\Gamma$. Without loss of generality we assume that $n_{0} \geq 3$ (see above). Then the groups $\operatorname{SL}\left(n_{0}, q_{0}\right)$ and $\operatorname{Sp}\left(n_{0}, q_{0}\right)$ act transitively on the set $V^{*}$ [5, Lemma 2.10.5]. By the intransitivity of $\Gamma$ this implies that $H_{0}$ cannot be one of these groups. Therefore we may assume that $H_{0}$ is either the unitary group $\operatorname{SU}\left(n_{0}, q_{0}^{1 / 2}\right)$ or the orthogonal group $\Omega^{\epsilon}\left(n_{0}, q_{0}\right)$. Given an element $\lambda$ of the ground field $\mathbb{F}$, set

$$
V_{\lambda}^{*}= \begin{cases}\left\{v \in V^{*}: f(v, v)=\lambda\right\} & \text { if } H_{0}=\operatorname{SU}\left(n_{0}, q_{0}^{1 / 2}\right) \\ \left\{v \in V^{*}: Q(v)=\lambda\right\} & \text { if } H_{0}=\Omega^{\epsilon}\left(n_{0}, q_{0}\right)\end{cases}
$$

where $f$ (resp. $Q$ ) is the nondegenerate unitary (resp. quadratic) form corresponding to $H_{0}$. By the same lemma, for $n_{0} \geq 3$, we obtain that

$$
\operatorname{Orb}\left(H_{0}, V^{*}\right)=\left\{V_{\lambda}^{*}: \lambda \in \mathbb{F}\right\} \quad \text { unless } n_{0}=3, H_{0}=\Omega\left(3, q_{0}\right), \lambda=0,
$$

and in the exceptional case the set $V_{\lambda}^{*}$ is the union of two $H_{0}$-orbits each of size $\left(q_{0}^{2}-1\right) / 2$. Clearly, the number $a_{\lambda}=\left|V_{\lambda}^{*}\right|$ does not depend on $\lambda \neq 0$. So using the explicit formulas for $a_{0}$ (see Lemma 10.4 and Theorem 11.5 in [12]), one can see that $q_{0}$ is coprime to $a_{0}$ and divides $a_{\lambda}$ for all $\lambda \neq 0$. Since $\Gamma$ acts on the set $\operatorname{Orb}\left(H_{0}, V^{*}\right)$ (due to the normality of $H_{0}$ in $\Gamma$ ), this implies that

$$
\left(V_{0}^{*}\right)^{\Gamma}=V_{0}^{*} \text { and }\left|\operatorname{Orb}\left(\Gamma, V_{0}^{*}\right)\right| \leq 2
$$

By the half-transitivity of $\Gamma$ this shows that the size of any $X \in \operatorname{Orb}\left(\Gamma, V^{*}\right)$ is coprime to $q_{0}$. However, this contradicts the fact that $q_{0}$ divides $|X|$ for all $X \not \subset V_{0}^{*}$, and we are done. 
Proof of Theorem 1.3 The hypothesis shows that $r$ divides the order $m$ of the base group of the scheme $\mathcal{C}$. So this group contains a cyclic subgroup $G$ of order $r$. By Theorem 1.1 we have

$$
G \leq \Gamma \leq \mathrm{GL}(k, p),
$$

where $\Gamma=\operatorname{Aut}(\mathcal{C})_{v}$ with $v=0$. Besides, the orbits in the action of $\Gamma$ on $V^{*}$ all have the same size $m<p^{k}-1$, and hence the group $\Gamma$ is half-transitive. Thus by Theorem 4.1 with $(q, n)=(p, k)$ it suffices to check only the cases $(p, k) \in\{(2,4),(2,6)\}$. However, the fact that $\left(2^{d}, n\right)$ with $d n \in\{4,6\}$ is a Dickson pair implies that either $(d, n)=(2,3)$ or $n=1$. But, in the former case $k=6$ and $Z_{2 k+1}(2, k)=\emptyset$, whereas in the latter case the near-field is a field, and we are done (see Sect. 1).

The following auxiliary lemma is a combination of some number theoretical results from [10] and [11]; it will be used in the proof of Theorem 1.4.

Lemma 4.2 Given a prime power $q=p^{d}$, there exists an integer $N_{q} \in \mathbb{N}$ such that the set $Z_{2 d n+1}(p, d n)$ is not empty for all $n>N_{q}$.

Proof Given $n \in \mathbb{N}$, denote by $D(n)$ the number of distinct prime factors of $n$, by $P[n]$ the greatest of them, and by $\Phi_{n}(X)$ the cyclotomic polynomial of degree $\varphi(n)$. Then there exists a constant $C>0$ such that

$$
P\left[\Phi_{d n}(p)\right]>C n \sqrt{\log n} / \log \log \log n
$$

for all sufficiently large $n$ such that $D(d n) \leq \kappa \log \log (d n)$ with $\kappa=1 /(2 \log 2)$ (see $[10$, p. 25]).

Denote by $S_{q}$ the set of all integers $d n \in N$ such that $(q, n)$ is a Dickson pair and $n$ is greater than the minimal number $a \in \mathbb{N}$ for which $D(d) \log q \leq \kappa \log \log (d a)$. Then given $d n \in S_{q}$, we have

$$
D(d n) \leq D(d) D(n) \leq D(d) \log q \leq \kappa \log \log (d n) .
$$

By (10) this implies that $P\left[\Phi_{d n}(p)\right]>2 d n+1$ for all sufficiently large $n \in S_{q}$. However, a prime factor $r$ of the number $\Phi_{d n}(p)$ is not a Zsigmondy prime for $(p, d n)$ iff $r \leq d n$ (see [11, Proposition 2]). Thus there exists a positive integer $N_{q}$ such that $Z_{2 d n+1}(p, d n) \neq \emptyset$ for all $n>N_{q}$.

Proof of Theorem 1.4 Let $\mathcal{C}=\operatorname{Cyc}\left(K, \mathbb{K}^{\times}\right)$, where $\mathbb{K}$ is a Dickson near-field corresponding to the Dickson pair $(q, n)$, and the group $K \leq \mathbb{K}^{\times}$is of order $m<q^{n}$. Suppose that $n>N_{q}$. Then by Lemma 4.2 the set $Z_{2 d n+1}(p, d n)$ is not empty. Set $K^{\prime}$ to be a maximal subgroup of $\mathbb{K}^{\times}$containing $K$. Since the group $\mathbb{K}^{\times} \leq \Gamma \mathrm{L}\left(1, q^{n}\right)$ is supersolvable, the number $\left[\mathbb{K}^{\times}: K^{\prime}\right]$ is prime. So if $\left|Z_{2 d n+1}(p, d n)\right| \neq 1$, then the number $m^{\prime}=\left|K^{\prime}\right|$ has a prime divisor $r^{\prime} \in Z_{2 d n+1}(p, d n)$. This implies that the scheme $\mathcal{C}^{\prime}=\operatorname{Cyc}\left(K^{\prime}, \mathbb{K}^{\times}\right)$belongs to the class $\operatorname{Cyc}\left(q, n, m^{\prime}\right)$ and satisfies the hypothesis of Theorem 1.3 with $r$ replaced by $r^{\prime}$. Thus

$$
\operatorname{Aut}(\mathcal{C}) \leq \operatorname{Aut}\left(\mathcal{C}^{\prime}\right) \leq \operatorname{A\Gamma L}\left(1, q^{n}\right)
$$

and we are done. 


\section{References}

1. Brouwer, A. E., Cohen, A. M., \& Neumaier, A. (1989). Distance-regular graphs. Berlin: Springer.

2. Cossidente, A., \& de Resmini, M. J. (2004). Remarks on Singer cyclic groups and their normalizers. Designs, Codes and Cryptography, 32, 97-102.

3. Dixon, J. D., \& Mortimer, B. (1996). Permutation groups. Graduate texts in mathematics (Vol. 163). New York: Springer.

4. Guralnick, R., Penttila, T., Praeger, C. E., \& Saxl, J. (1999). Linear groups with orders having certain large prime divisors. Proceedings of the London Mathematical Society, 78, 167-214.

5. Kleidman, P., \& Liebeck, M. (1990). The subgroup structure of the finite classical groups. London mathematical society lecture note series (Vol. 129). Cambridge: Cambridge University Press.

6. Manz, O., \& Wolf, T. R. (1993). Representations of solvable groups. London mathematical society lecture note series (Vol. 185). Cambridge: Cambridge University Press.

7. Neumann, P. M., \& Praeger, C. E. (1992). A recognition algorithm for special linear groups. Proceedings of the London Mathematical Society, 65, 555-603.

8. Passman, D. S. (1968). p-Solvable doubly transitive permutation groups. Pacific Journal of Mathematics, 26, 555-577.

9. Praeger, C. E., \& Saxl, J. (1992). Closures of finite primitive permutation groups. The Bulletin of the London Mathematical Society, 24, 251-258.

10. Ribenboim, P. (2000). My numbers, my friends. Popular lectures on number theory. New York: Springer.

11. Roitman, M. (1997). On Zsigmondy primes. Proceedings of the American Mathematical Society, 125, 1913-1919.

12. Taylor, D. E. (1992). The geometry of the classical groups. Berlin: Heldermann.

13. Wähling, H. (1987). Theorie der Fastkörper. Essen: Thales.

14. Wielandt, H. (1964). Finite permutation groups. New York: Academic. 